\title{
Moment Generating Function Approach in Diversity Combiner with M-PSK over Hoyt Fading Channel
}

\author{
Adeyemo Z.K, Ajadi, A.S., Semire, F.A., Ojo, S.I. \\ Department of Electronic and Electrical Engineering, \\ Ladoke Akintola University of Technology, Ogbomoso, Oyo State, Nigeria. \\ zkadeyemo@lautech.edu.ng
}

\begin{abstract}
Wireless communication system is the processing, transmitting and receiving signals over an open space. This system suffers from time varying environment disturbance due to propagation of signals through different paths which prompt the signals to have different statistical distributions. The existing modified diversity combiners diversity such as Maximal Ratio Combiner (MRC), Equal Gain Combiner (EGC) with one Match Filter (MF) and one Radio Frequency (RF) chain, lack closed form expression. Therefore, in this paper, Moment Generating Function (MGF) approach is used to analyse the performance of the modified MRC over Hoyt fading distribution through the closed form expression. A closed formed expression is developed using the existing modified MRC with one Radio Frequency (RF) chain and one Matched Filter (MF) at the RF stage. Binary data of ten thousand bits are generated randomly as source data and modulated with M-ary Phase Shift Keying (M-PSK). The modulated signal is passed through the Hoyt fading channel which is then modeled using Moment Generating Function (MGF) approach in order to generate the resultant signals. The resultant signals at varying paths ' $L$ ' (2, 3 and 4$)$ and Hoyt fading factors' $q$ ' (0.2, 0.4 and 0.6 ) are combined using the modified MRC. The output signal is passed through a comparator which compares the output signal with $9.5 \mathrm{~dB}$ set as threshold value. The outage probability of the modified MRC using M-PSK (2-PSK and 4-PSK) at varying ' $L$ ' and ' $q$ ' is evaluated, while processing time is used to compare the performance of the conventional MRC with the modified MRC. The results obtained with the modified MRC using closed form expression give lower Outage Probability (OP) and processing time. The research can be used by satellite communication system designers to improve the quality of service.
\end{abstract}

Keywords: Radio Frequency (RF), Moment Generating Function (MGF), Maximum Ratio Combiner (MRC), M-PSK, Match Filter (MF)

\section{Introduction}

Wireless communication is the transfer of information between two or more locations that are not connected by an electrical conductor. Wireless communication systems that have been deployed are first, second and third generations. The fourth and fifth generations systems are currently under deployment in some developing countries. The first generation wireless systems are analog in nature and make use of Frequency Division Multiple Access (FDMA) as multiple access technology. The second generating systems are digital in nature and use Time Division Multiple Access (TDMA) or Code Division Multiple 
Adeyemo Z.K, Ajadi, A.S., Semire, F.A., Ojo, S.I.; Moment Generating Function Approach in Diversity Combiner with MPSK over Hoyt Fading Channel, Transactions on Networks and Communications, Volume 7 No. 1, February (2019); pp: $51-62$

Access (CDMA) as multiple access scheme. This has some features like data encryption, authentication, automatic location services and so on. The third generation systems are also digital in nature and are equipped with infrastructure to support Personal Communications Systems (PCS) such as Low Earth Orbit (LEO), satellite networks, wireless Asynchronous Transfer Mode (ATM) networks, mobile Internet Protocol (IP) and so on. Fourth and fifth generation systems have enhanced features over the third generation such as higher spectral efficiency, higher data rates, downlink speed of $100 \mathrm{Mbps}$ and uplink of 50Mbps. They use multicarrier modulation especially Orthogonal Frequency Division Multiple Access (OFDMA) and Multiple antenna that is Multiple Input Multiple Output (MIMO) to achieve the requirement of the system, but these are currently being deployed. Wireless technologies according to [30] use radio waves to make the distance between the transmitter and receiver appears to be short.

Signal transmitted through the wireless channels suffers from time varying channel which makes the received signal unpredictable due to fluctuations. This phenomenon is known as fading, which may vary with time, geographical location, or radio frequency, and is often modeled as a random process [15, 25, $21,29]$. Fading in wireless communication may either be due to multipath propagation referred to as multipath fading or to shadowing from obstacles affecting the signal propagation $[2,3,10,12]$. Multipath propagation is a phenomenon that results in radio signals reaching the receiving antenna through two or more paths, which are due to atmospheric ducting, reflection, refraction, mountains and buildings. The multipath propagation effects are interference, signal delay and phase shifting of the signal. In wireless communication, multipath causes error and affects the quality of the received signal. It can be modeled statistically using different fading distributions such as Rayleigh, Rician, Nakagami, Weibull and Lognormal fading distributions $[10,11,13,17,22,24]$.

Nakagami distribution is of different forms namely: Nakagami $q$, Nakagami $m$ and so on. Nakagami-q is also known as Hoyt distribution, this is being used in describing the short term signal variation of wireless communication systems [29]. Hoyt channel model has been statistically used in modeling of satellite communication. The faded signal through Hoyt fading channel can be processed by different mitigating techniques such as diversity technique, equalization technique and so on. Diversity technique has gained popularity in combating the effects of fading at the receiver. The basic principle of diversity is to create multiple copies of uncorrelated signals and combine them in an optimum way. Diversity can be classified as: space, frequency, time and polarization $[9,33,31]$.

However, multiple signal copies must be uncorrelated or weakly correlated for diversity combining to be most effective. Hence, different types of diversity are normally efficient in different scenarios. The most widely used techniques are namely: Selection Combining (SC), Maximal Ratio Combining (MRC), Equal Gain Combining (EGC), and Hybrid Combining [26, 28, 20, 23, 18]. It is a known fact that Maximal Ratio Combiner (MRC) provides better performance than all other diversity combining techniques in multipath environment. However, this conventional MRC has the highest complexity of all combining techniques due to many hardwares involved. Modified Maximal Ratio Combiner which will have a reduced complexity relative to the conventional MRC scheme is being researched into, but lacks a closed form expression. Maximal Ratio Combiner outperforms others but at the expense of hardware complexity [28, 34].

This paper addresses the challenges of hardware complexity and closed form expression posed by the modified MRC with single MF and RF chain in Hoyt fading channel. Although, there are commonly used performance metrics in wireless communication namely: Signal-to-Noise Ratio (SNR), Average Outage 
Duration (AOD), Bit Error Rate (BER), Amount of Fading (AF), and Outage Probability (Pout) [4]. However, Outage Probability which is the instantaneous error of probability that exceeds a specified value according to Goldsmith (2005) is employed in this paper for evaluating the performance.

\section{Wireless Communication Channel}

Signal transmission in wireless system is achieved through the inherent broadcast nature of electromagnetic waves. However, the electromagnetic waves are not guided to the receivers through some media in wireless communications. Therefore, in wireless communication systems, sending of radio signals is through the space, the signal radiated from the antenna reaches the receiver through different paths, this phenomenon which is known as multi-path fading in wireless communications, and dictates the quality of the received signal $[8,10,16]$. The limiting factors affecting the performance of radio propagation models are: path loss, shadowing, time dispersion, time variance, fading, interference and noise.

\subsection{Path Loss}

Path loss is the reduction in power density of an electromagnetic wave as it propagates through space. This phenomenon leads to signal attenuation [28]. According to [9], the path loss ' $P_{L}$ ' is given as:

$$
P_{L}=20 \log _{10}\left(\frac{4 \pi d}{\lambda}\right)
$$

where: $P_{L}$ is the path loss in decibels,

$\lambda$ is the wavelength,

$\mathrm{d}$ is the transmitter- receiver distance.

\subsection{Shadowing}

Shadowing is a random variable which varies according to types of environment where radio wave propagates and does not depend on the distance between the receiver and the transmitter. This is experienced due to absorption of radio waves in the propagation by scattering structures. In term of the quality, shadowing loss varies faster than the path loss, but it is slower than the fading $[24,27]$.

\subsection{Interference}

This is a distortion which disrupts a signal as it travels along a channel, it can be expressed as addition of unwanted signal to a useful signal. The source of interference in a radio system can be originated at the system itself or it can be located at external source. The two major types of interference are Inter-Symbol Interference (ISI) and Co-Channel Interference (CCI), [28, 32].

\subsection{Inter-Symbol Interference (ISI)}

This is a form of distortion in which one symbol interferes with subsequent symbols and thus, making the communication less reliable, $[28,32]$.

\subsection{Co-Channel Interference (CCI)}

This is the overlapping of signal unintentionally from different radio transmitters with the same frequency. There are several causes of co-channel radio interference, such as: adverse weather conditions, poor frequency planning and overcrowded of radio spectrum, [28, 32]. 
Adeyemo Z.K, Ajadi, A.S., Semire, F.A., Ojo, S.I.; Moment Generating Function Approach in Diversity Combiner with MPSK over Hoyt Fading Channel, Transactions on Networks and Communications, Volume 7 No. 1, February (2019); pp: $51-62$

\section{Statistical Representation of Fading Channel}

Multipath fading can be modeled with different statistical distributions in different environments. The commonly used statistical distributions in wireless communication are: Rayleigh, Rician and Nakagami distributions because of their close approximation to reality.

\subsection{Rayleigh Distribution}

In Rayleigh fading, channel model assumes that all the components that make up the resultant received signal are reflected or scattered and there is no direct path from the transmitter to the receiver. The Rayleigh distribution $\mathrm{P}_{\text {rayl }}(\mathrm{u})$ envelope of a received signal is given by Rappaport [28] as:

$$
P_{\text {rayl }}(\mathrm{u})=\frac{u}{\sigma^{2}} \exp -\left(\frac{r^{2}}{2 \sigma^{2}}\right) \quad 0 \leq \mathrm{u} \leq \infty
$$

where: $u$ is the amplitude of the received signal

$\sigma$ is the root mean square ( $\mathrm{rms}$ ) value of the received signal

$\sigma^{2}$ is the time-averaged power of the received signal

$2 \sigma^{2}$ is the pre-detection mean power of the received signal

\subsection{Rician Distribution}

Normally, there exists a dominant Line of Sight (LOS) path in addition to numerous diffused multipath components between the transmitter and receiver in micro-cellular environments. In such a case, the other faded signal components are superimposed on the dominant component and the resultant signal amplitude follows a Rician distribution, with the ratio between the LOS and diffused components denoted by the Rice factor $\mathrm{k}$ which is equal to the ratio of power of line of sight component to the average power of the scattered components. The complex envelope of the received signal $P_{\text {Rician }}(u)$ is given by [28] as

$$
P_{\text {Rician }}(u)=\frac{u}{\sigma^{2}} \exp -\left(\frac{u^{2}+A^{2}}{2 \sigma^{2}}\right) I_{o}\left(\frac{A u}{\sigma^{2}}\right) \quad u \geq 0
$$

where: $A$ is the peak amplitude of the dominant component,

Io $\left(\frac{A u}{\sigma^{2}}\right)$ denotes the modified Bessel function of the first land,

$u$ is the amplitude of the received signal.

$\frac{r^{2}}{2}$ is the instantaneous power

$\sigma$ is the standard deviation of the local power

The distribution is expressed in term of the Rice factor $\mathrm{k}=\mathrm{A} /\left(2 \sigma^{2}\right)$ which is the ratio of the dominant components power to the variance of the combined power of the multipath components. As the power in the dominant component decreases to zero, the Rician distribution can be shown to approach the Rayleigh distribution.

\subsection{Nakagami-m distribution}

It is possible to describe both Rayleigh and Rician distributions with the help of a fading channel model using the Nakagami distribution. Nakagami-m distribution often gives the best fit to land mobile and indoor mobile propagation, as well as scintillation of ionospheric radio links. It fits experimental data much better than a Rayleigh or Rician distribution [29, 31] The Nakagami-m distribution envelope of the received signal $P_{N a k}(u)$ is given by $[24,31]$ as 


$$
P_{N a k}(u)=\frac{2}{\Gamma(\mathrm{m})}\left(\frac{m}{2 \sigma^{2}}\right)^{m} u^{2 m-1} \exp \left(\frac{-m u^{2}}{2 \sigma^{2}}\right) \quad u \geq 0
$$

where: ' $\sigma$ ' is the received rms envelope level,

$\mathrm{m}$ is the fading severity index,

$\Gamma(\cdot)$ denotes the gamma function,

$u$ is the amplitude of the received signal

When $\mathrm{m}=1$, Nikagami distribution degenerates to a Rayleigh distribution and closely approximates the Rician distribution via relationship $m=(k=1)^{2} /(2 k=1),[24]$.

\subsection{Nakagami -q distribution}

The Nakagami - q distribution also refers to as Hoyt distribution is commonly used to describe a shortterm signal variation of certain wireless communication system subject to fading. Specifically, the Hoyt channel has been applied in satellite based cellular communications to characterize more severe fading condition than those modeled by Rayleigh. The Probability Density Function (PDF) of Nakagami-q distribution ' $P_{\text {Nakg' }}(u)$ is given by [4] as:

$$
P_{\text {Nakq }}(\mathrm{u})=\frac{u}{\sigma_{1} \sigma_{2}} \exp \left(\frac{u^{2}}{4}\left(\frac{1}{\sigma_{1}^{2}}-\frac{1}{\sigma_{2}^{2}}\right)\right) I_{o}\left(\frac{u^{2}}{4}\left(\sigma_{2}^{2}-\sigma_{1}^{2}\right)\right)
$$

where: $I_{0}($.) denotes the zeroth order modified based function of the first kind,

$u$ is the amplitude of the received signal.

$\sigma_{1}$ and $\sigma_{2}$ are zero mean and variance, respectively.

\section{$4 \quad$ MRC with Single MF and Single RF Chain}

Conventional MRC uses many RF chain and Match Filters (MFs) due to multiple copies of the transmitted signal resulting in high hardware complexity. But MRC with single MF and single RF chain makes use of only one RF chain and one MF [9]. This is performed by co-phasing and weighing the received signals through different branches before adding. This type of MRC is shown in Figure 1, where $H_{1}, H_{2} \ldots H_{L}$ represent the Hoyt fading through different paths over which the transmitted signals $S_{1}(t), S_{2}(t) \ldots S_{L}(t)$ propagate to the receiver. The faded signals are combined by MRC and passed through only one RF chain and one MF for reception.

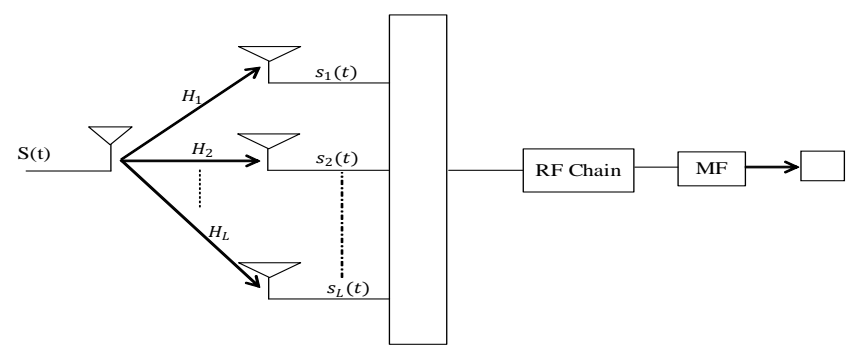

Fig.1: MRC with single MF and single RF chain

\section{Development of Closed Form Expression for System Model}

In signal transmitting over Hoyt fading channel, the baseband representation of the received signal ' $y$ ' is given by [29] as:

$$
\mathrm{y}=\mathrm{Xs}+\mathrm{n}
$$

where; ' $X$ ' is the channel fading envelop which is Hoyt distributed

' $s$ ' is the transmitted baseband signal and

' $n$ ' is the Additive White Gaussian Noise (AWGN) 
Adeyemo Z.K, Ajadi, A.S., Semire, F.A., Ojo, S.I.; Moment Generating Function Approach in Diversity Combiner with MPSK over Hoyt Fading Channel, Transactions on Networks and Communications, Volume 7 No. 1, February (2019); pp: 51-62

The receiver weights all the inputs signals separately and sums them to make a decision. The transmitted symbols of the instantaneous output SNR per symbol ' $\gamma$ ' is expressed by [29] as

$$
\gamma=\frac{E_{b}}{N_{o}} \alpha^{2}
$$

Mathematically, instantaneous output from MRC $\gamma_{M R C}$ can be expressed as:

$$
\gamma_{M R C}=\frac{E_{b}}{N_{o}}\left(\alpha_{1}^{2}+\alpha_{2}^{2}+\cdots+\alpha_{L}^{2}\right)=\frac{E_{b}}{N_{o}} \alpha^{2}
$$

where; $\alpha^{2}=\left(\alpha^{2}{ }_{1}+\alpha^{2}{ }_{2}+\cdots+\alpha^{2}{ }_{L}\right)$, which means that the PDF of $\gamma_{M R C}$ can be obtained from the PDF of the Random Variable RV $\alpha^{2}$.

The Nakagami-q (q being the fading severity parameter) distribution is generally used to characterize the environment that is more severe than Rayleigh fading. The corresponding PDF of the fading envelope $f \alpha(\alpha)$ is given by [29] as:

$$
f \alpha(\alpha)=\frac{\left(1+q^{2}\right) \alpha}{q \Omega} \exp \left[-\frac{\left(1+q^{2}\right)^{2} \alpha^{2}}{4 q^{2} \Omega}\right] \text { lo }\left[\frac{\left(1-q^{4}\right) \alpha^{2}}{4 q^{2} \Omega}\right] ; \alpha \geq 0
$$

where $\Omega=\mathrm{E}\left[\alpha^{2}\right]$ and $\mathrm{Io}($.$) denotes zeroth order modified Bessel function of first kind.$

According to [29], the SNR per symbol of the channel $f_{\gamma}(\gamma)$ is distributed as

$$
f_{\gamma}(\gamma)=\frac{\left(1+q^{2}\right) \alpha}{2 q \gamma_{a}} \exp \left[-\frac{\left(1+q^{2}\right)^{2} \gamma^{2}}{4 q^{2} \gamma_{a}}\right] \text { lo }\left[\frac{\left(1-q^{4}\right) \gamma}{4 q^{2} \gamma_{a}}\right] ; \gamma \geq 0
$$

Finding the $\mathrm{k}^{\text {th }}$ moment using (10), the indefinite integrals involving exponential and Bessel functions are solved using Marcum's $Q$ function and the closed form expression of $\mathrm{k}^{\mathrm{th}}$ moment of $\gamma$ output SNR obtained as:

$$
E\left(\gamma^{k}\right)=\Gamma(1+k)_{2} \mathrm{~F}_{1}\left(-\frac{k-1}{2}, \frac{-k}{2} ; 1,\left(\frac{1-q^{2}}{1+q^{2}}\right)^{2}\right) \gamma_{a}^{k}
$$

In order to quantify the performance in term of OP, MGF based approach is used with Pade Approximation (PA) technique to find simple way of evaluating rational expression for the MGF.

\subsection{Moment Generating Function (MGF) of the SNR Output}

The MGF $M_{\gamma}(s)$ is the Laplace transform of the PDF of a distribution and is given by $[1,29]$ as

$$
M_{\gamma}(s)=E\left(\mathrm{e}^{s \gamma}\right)=\int_{0}^{\infty} \mathrm{e}^{s \gamma} P_{\gamma}(\gamma) d \gamma \quad \gamma>0
$$

By expanding $\left(\mathrm{e}^{\mathrm{s} \gamma}\right)$ in equation (12), results in

$$
\mathrm{M}_{\gamma}(\mathrm{s})=\sum_{k=0}^{\infty} \frac{s^{k} \sum\left(\gamma^{k}\right)}{k !}=\sum_{k=0}^{\infty} \frac{s^{k} H_{k}}{k !}
$$

where $\sum\left(\gamma^{k}\right)=\mathrm{H}_{\mathrm{k}}$ and is equal to the $\mathrm{k}^{\mathrm{th}}$ moment represented by equation (12)

$$
M_{\gamma}(s)=\frac{(-1)^{k} \Gamma(1+k)}{k !}{ }_{2} \mathrm{~F}_{1}\left(-\frac{k-1}{2}, \frac{-k}{2} ; 1,\left(\frac{1-q^{2}}{1+q^{2}}\right)^{2}\right) X^{\mathrm{k}} \text { where } X=s \gamma_{a}
$$

when $k=0$ in 3.10, the coefficient of the expression becomes one. Using MATLAB 7.0 software when $q=0: 0.2: 1$, k ranges from 0 to 19 . 


\section{Padé Approximants (PA) of the MGF of SNR Output}

Employing the work of $[6,8]$ a PA which is a rational function approximation of a power series is used to rationalize equation 3.12 in order to obtain its inverse Laplace transform. In this case, $M_{\gamma}(s)$ has a specified order $\mathrm{B}$ for the denominator and $\mathrm{A}$ for the numerator, where $\mathrm{A}$ and $\mathrm{B}$ are positive integers [1].

$$
R(x)=\frac{\sum_{i=0}^{A} a_{i} x^{i}}{1+\sum_{j=0}^{B} b_{j} x^{j}}=M_{\gamma}(s)=\sum_{n=0}^{N} C_{n} x^{k}
$$

where $C_{n}$ are the coefficients of equation (16), $\mathrm{B}=\mathrm{A}+1$. This implies that order of denominator is greater than that of the numerator by $1 . a_{i}$ and $b_{j}$ are real coefficients which are determined by solving the set of $\mathrm{A}+\mathrm{B}+1$ equations, with an assumption that $b_{0}=1$

$$
\sum_{j=0}^{B} b_{j} c_{B-1-j+l}=00 \leq l \leq B
$$

After solving for the values of $b_{j}$, the set $a_{i}$ is obtained through back substitution from,

$$
a_{i}=c_{i}+\sum_{p=1}^{\min (B, i)} b_{i} c_{i-p}=00 \leq l \leq A
$$

MATLAB was used to generate the coefficients $a_{i}$ and $b_{j}$. The Hankel matrix rank deficient above $\mathrm{N}=15$. and the PA of equation (16) is given in equation.

$$
R(x)=\frac{1+7.0528 x+22.1531 x^{2}+40.4388 x^{3}+46.8885 x^{4}+35.3433 x^{5}+16.9064 x^{6}+4.6910 x^{7}+0.5779 x^{8}}{1+7.921 x+28.5288 x^{2}+60.7805 x^{3}+845825 x^{4}+79.6900 x^{5}+50.8123 x^{6}+21.1380 x^{7}+5.2049 x^{8}+0.5779 x^{9}}
$$

\subsection{Outage probability of MRC Output}

The signal outage probability is defined as the probability that the instantaneous SNR will fall below a certain threshold.

$$
\left.\operatorname{Pout}\left(\gamma_{t h}\right)=\operatorname{Pr}(S N R) \leq \gamma_{t h}\right)
$$

For MRC receiver with L identical and independently distributed channels, the signal outage probability is given by

$$
\text { PMRC_out }\left(\gamma_{t h}\right)=\frac{1}{2 \pi j} \int_{\varepsilon-j \alpha}^{\varepsilon+j \alpha} \frac{\left[M_{\gamma}(S)\right]^{L}}{S} e^{S \gamma_{t h}} \mathrm{ds}
$$

where $\varepsilon$ is a properly chosen constant in the region of convergence of complex s-plane. Since $M_{\gamma}(s)$ is in term of rational function, it is, therefore, possible to use partial fraction expression of $\frac{\left[M_{\gamma}(s)\right]^{L}}{s}$ in (21) to evaluate outage probability, meaning

$$
\begin{gathered}
\mathrm{P}_{\text {out }}\left(\gamma_{t h}\right)=\frac{1}{2 \pi j} \int_{\mathcal{E}-j \alpha}^{\varepsilon+j \alpha} \sum_{i=1}^{N_{p}} \frac{\lambda_{i}}{S+P_{i}} e^{S \gamma_{t h} \mathrm{ds}} \\
=\frac{1}{2 \pi j} \sum_{i=1}^{N_{p}} \int_{\mathcal{E}-j \alpha}^{\varepsilon+j \alpha} \frac{\lambda_{i}}{S+P_{i}} e^{S \gamma_{t h} \mathrm{ds}} \\
=\sum_{i=1}^{N_{p}} \lambda_{i} e^{P_{i} \gamma_{t h}}
\end{gathered}
$$

where $\mathrm{p}_{\mathrm{i}}$ are $N_{p}$ poles of rational function in ' $\mathrm{s}$ ' with ' $\lambda_{i}$ ' its residues. Each term inside the summation in (8) represents a simple rational function form. 
Adeyemo Z.K, Ajadi, A.S., Semire, F.A., Ojo, S.I.; Moment Generating Function Approach in Diversity Combiner with MPSK over Hoyt Fading Channel, Transactions on Networks and Communications, Volume 7 No. 1, February (2019); pp: $51-62$

\section{Development of the System Simulation Model}

The system model consists of a transmitter to process the randomly generated data which is available within the MATLAB simulation software for transmission by converting the source data into bits, reshaping and modulating with M-PSK signaling scheme. The Square-Root Raised Cosine (SRRC) filter is used at both transmitter and receiver to reduce the effect of the Inter Symbol Interference (ISI) and the match filtering criterion to maximize SNR of the system. The acquired data is processed using modified Maximal Ratio Combining technique. The complete system model for this work is shown in Figure 2 . The system model is simulated using MATLAB simulation software with some simulation parameters contained in Table 1. in accordance with the wireless standards. The Outage probability is calculated by equating the threshold value to be $9.5 \mathrm{~dB}$.

Table 1: System simulation parameters for modified MRC

\begin{tabular}{ll}
\hline Parameter & Specification \\
\hline Modulation schemes & M-PSK \\
Fading & Hoyt \\
Number of MRC paths & $3,4,5$ \\
Carrier Frequency & $1800 \mathrm{MHz}$ \\
Bandwidth of symbol & $250 \mathrm{kHz}$ \\
Delay spread & $250 \mathrm{~ns}$ \\
Noise & AWGN \\
Transmit Filter & Square Root Raised Cosine \\
Receiver Filter & Square Root Raised Cosine \\
Roll of factor & 0.25 \\
Number of samples/symbol & 10 \\
SNR & $0: 2: 12$ \\
Number of symbol (data length) & 10,000 \\
\hline
\end{tabular}

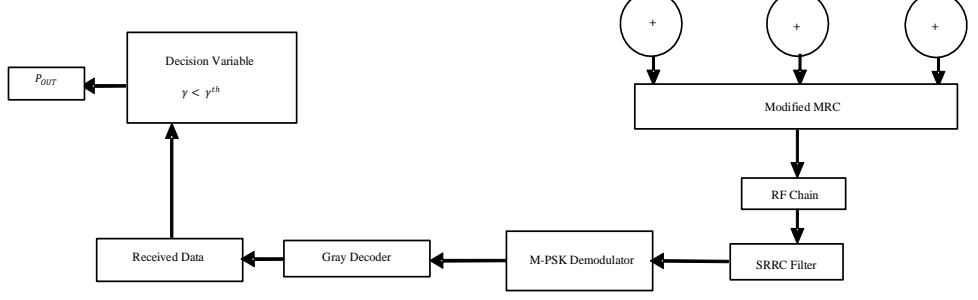

Figure 2: The Developed System Simulation Model.

\section{Results and Discussion}

The results obtained for the modified MRC at varying paths ' $L$ ' $(2,3,4)$ and different Hoyt fading factors-q $0.2,0.4$ and 0.6 using M-PSK signaling schemes are presented in Figures. 3-11. Figure 3 shows the OP values versus Signal to Noise Ratio for the modified MRC for varying ' $L$ ' at $q=0.2$ using Binary Phase Shift Keying (BPSK) scheme. The OP values obtained at the threshold value of $9.5 \mathrm{~dB}$ for $\mathrm{L}=2,3$ and 4 are 0.1499 , 0.1354 and 0.1217 , respectively. Also, Figure 4 depicts the simulated OP versus signal to Noise Ratio of 
the modified MRC at $q=0.4$ using 2-PSK in which OP values obtained at $9.8 \mathrm{~dB}$ are $0.99001,0.97111$, 0.90017 for L=2, 3 and 4, respectively. Similarly, Figure 5 shows the OP versus Signal to Noise Ratio of the modified $\mathrm{MRC}$ at $\mathrm{q}=0.6$ using 2-PSK signalling scheme. It can be confirmed from Figure 5 that the OP values obtained are $0.97111,0.95002$ and 0.90001 for $L=2,3$ and 4, respectively, while Figure 6 reveals the OP versus SNR of the modified MRC at $\mathrm{q}=0.2$ using 4-PSK signaling scheme known as Quadrature Shift Keying (QPSK) scheme. The OP values obtained for Figure 6 at SNR of $9.8 \mathrm{~dB}$ are $0.9900,0.9982$ and 0.98223 for $\mathrm{L}=2,3,4$, respectively. Table 2 contains the processing time of the conventional MRC and the modified MRC at $q=0.2$ with 2-PSK (BPSK) scheme. It can be confirmed that the processing time (s) at $q=0.2$ for 2-PSK (BPSK) scheme for conventional MRC are 3.88527, 4.72021 and 5.470033s for ' $L$ ' $=2,3$ and 4, respectively, while the processing time at $q=0.2$ with 2-PSK for the modified MRC are $0.744267,1.503282$ and 2.18500 for $L=2,3$ and 4 , respectively. The results obtained with the modified MRC using closed form expression give lower OP and processing time. The results are justifiable in that only one RF chain and one MF are used at RF stage, thereby reducing the hardware complexity as a result of reduction of processing time. It is also observed that as the number of paths ' $\mathrm{L}$ ' increases, the OP decreases while the processing time increases, thereby improving the performance. It can also be deduced as Hoyt fading factor ' $q$ ' increases indicating decreases in severity of fading resulting in decrease in probability of outage. The result could be used by satellite communication system.

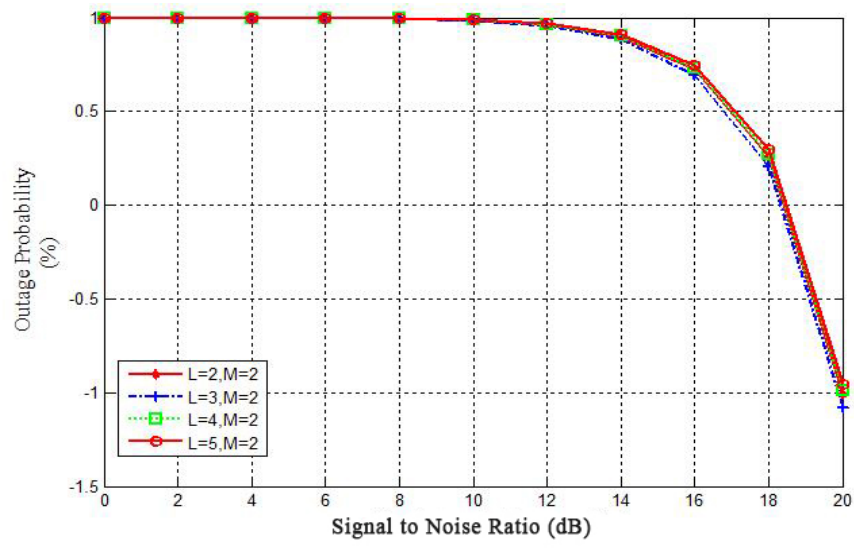

Figure 3: Simulated Pout of 2-PSK (BPSK) versus Signal-to-Noise Ratio (dB) with the Modified MRC for Varying $\mathrm{L}$ at $\mathrm{q}=\mathbf{0 . 2}$.

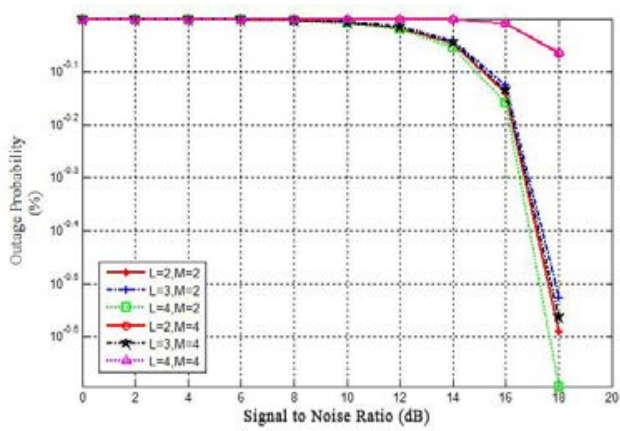

Fig. 4: Simulated Pout versus Signal-to-Noise Ratio (dB) with the Modified MRC for Varying ' $L$ ' and $M$ at $q=0.4$. 


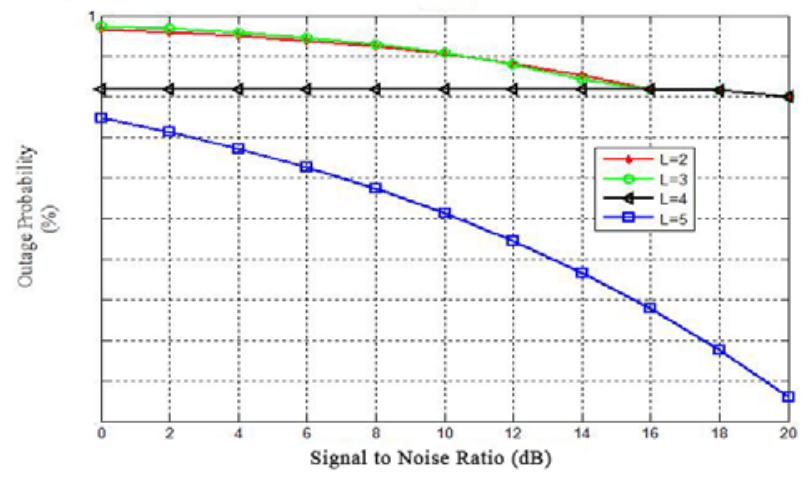

Figure 5: Outage Probability versus Signal-to-Noise Ratio (dB) with Modified MRC for Varying ' $L$ ' at $q=0.6$

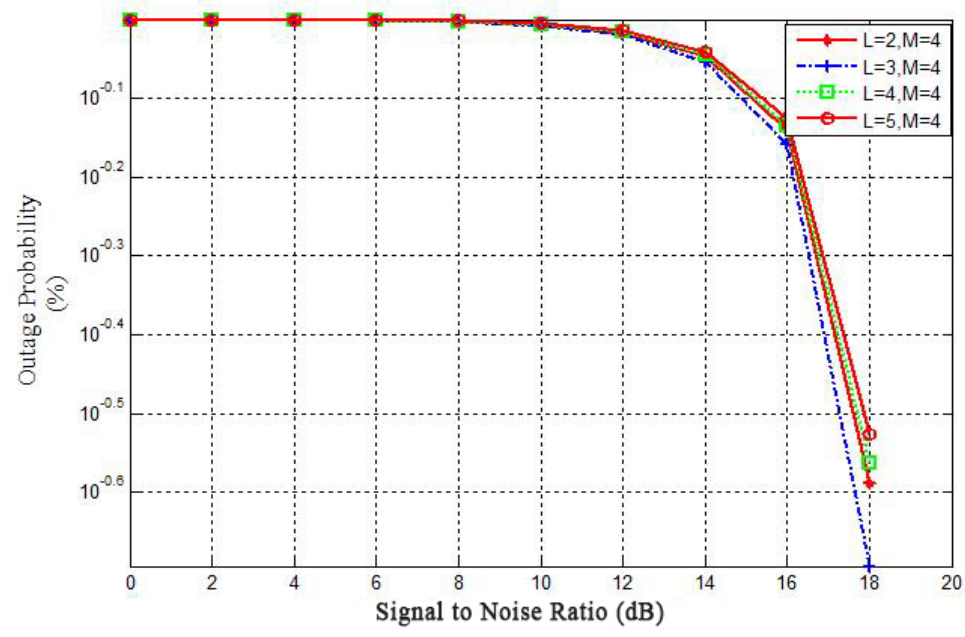

Figure 6: Simulated Pout of 4-PSK (QPSK) versus Signal-to-Noise Ratio (dB) with the Modified MRC for Varying $\mathrm{L}$ at $\mathrm{q}=0.2$

\section{Conclusion}

The Moment Generating Function (MGF) approach in MRC with single MF and single RF chain using MPSK over Hoyt fading channel has been developed. The modified MRC with single RF chain and single MF has been investigated over Hoyt fading channel. The closed form expression for the modified MRC over different Hoyt fading factors using MGF approach has been developed for various paths ' $L$ ' $(2,3,4)$. The developed channel model has been incorporated in the system model using M-PSK signaling scheme at different constellations. The system model has been simulated using MATLAB simulation software and evaluated using outage probability. It has been shown that the modified MRC using closed form expression gives lower outage probability and processing time.

\section{REFERENCES}

[1] Abramovitz, M., and Stegun, I.A, "Hand book of Mathematical functions with formulas, graphs, and mathematical tables" Dover, New York, USA. (1992),

[2] Adeyemo, Z. K. and Raji T. I, "Effects of Diversity combining in mobile Terrestrial Environment" Continental Journal of Engineering Sciences 5:27-37. (2010) 
[3] Adeyemo, Z. K. and Ojedokun Isaac A., "EGC Receiver using single Radio Frequency chain and single matched filter over combined Rayleigh and Rician Fading channels" ARPN Journal of Engineering and Applied Science, (2014). 9(7): 1819-6608.

[4] Anamllyas, Ejaz Ansari and Saleem Akhtar "Accurate BER\SER Analysis and performance of different modulation schemes over wireless fading channel," Science Inernation, (2013). 25(2):367-374.

[5] Adeyemo Z.K, Rabiu E.O and Abolade R.O. Offset Phase Shift Keying modulation in Multiple-Input MultipleOutput Spatial Multiplexing; Transaction on Networks and Communications, (2015): 3(20): 117-127.

[6] Amindavar H., and Ritcey J. A., "Pade approximation of probability density functions", IEEE Transaction Aerospace. Electron System, (1994). 30(1):416-424

[7] Annamalai A., and Tellambura C., "Performance evaluation of generalized selection Diversity systems over Nakagami-m fading channels", Wireless Communications and Mobile Computing, (2003). 3(1):99 - 116.

[8] Blaunstein, N., and Christodoulou, C. G., "Radio Propagation and Adaptive Antennas for Wireless Communication Links", John Wiley and Sons, Inc., Hoboken, New Jersey. (2007),

[9] Brennan, D.G, “Linear Diversity Combining Techniques," Proceeding of the IEEE, (2003), 91 (2):1075-1102

[10] Dighe, P. A., Mallik, R. K., and Jamuar, S. S., “Analysis of transmit-receive diversity in Rayleigh Fading", IEEE Transactions on communication, (2003). 51(1):694 - 703.

[11] Dimitris A. Zogas "Equal Gain Combining over Nakagami-n (Rice) and Nakagamiq(Hoyt) Generalized fading channels" IEEE Transactions on wireless communications, (2005). 4(2): $374-379$

[12] Goldsmith, A., "Wireless Communications," Standford University Press, California. (2005),

[13] Helstrom C.W., "Probability and Stochastic processes for engineers", Second edition Macmillan, New York, USA. (1991),

[14] Hoyt, R., "Probability functions for the modulus and angle of the normal complex variate," Belly Syst. Tech. J., (1947). 26(1):318-359.

[15] Jahn, A., "Propagation Considerations and fading counter measures for Mobile Multimedia Services", Int. Journal of Satellite Communication, (2001). 19(3), pp 223-250

[16] Jochen S., Mobile Communications, $2^{\text {nd }}$ Ed. Pearson Education Ltd., India. (2006).

[17] Jyoteesh Malhotra, Ajay Sharma, K., and Kaler R.S., "On the performance analysis of wireless receiver using generalized- gamma fading model”, Annals of Telecommunications. (2009). 46(1) :147-157.

[18] Karagiannidis G. K., Sagias N. C., and Zogas D. A. "Error analysis of M-QAM with equal Gain Combining diversity over generalized fading channels", IEEE Transactions on wireless communications, (2005). 152(1):69-74.

[19] Kim S.W. and Wang Z. "Maximum Ratio Diversity Combining Receiver using single Radio Frequency chain and single Matched Filter", IEEE Globecom proceedings, (2007). 2(1):269-274.

[20] Lee T., and Lee Z “A Beam Space Diversity Combiner for sector Division Multiple Access Communications”, IEEE Antennas and Propagation Society Symposium Digest, Atlanta, (1998) 1(1):372 - 375. 
Adeyemo Z.K, Ajadi, A.S., Semire, F.A., Ojo, S.I.; Moment Generating Function Approach in Diversity Combiner with M-

PSK over Hoyt Fading Channel, Transactions on Networks and Communications, Volume 7 No. 1, February (2019); pp: 51-62

[21] Malhotra J. "Investigation of M-QAM and MPSK with EGC in Generalized Flat Fading channels", Journal of Advances in information Technology, (2011). 2(4):250-256.

[22] Malhotra J., Sharma A. K., and Kaler R. S. "On the performance Analysis of wireless receiver using generalized-gamma fading Model”, Annals of Telecom, (2009), Vol. 64, No. 1, pp147-153.

[23] Mohammed J., Leszek S., and Mustapha B., "Outage probability of Diversity combining Receivers in Arbitrarily Fading channels, (2011)

[24] Nakagami M., "The m-distribution- A General Formula of intensity Distribution of Rapid Fading" Statistical Methods of Radio Wave Propagation, pergamon (1960). 1(1):3-36.

[25] Patzoid, M., "Mobile Fading Channels" John Wiley and Sons, Ltd, Baffins Lane, Chichester, West Sussex, po191 IUD, England. (2002).

[26] Pornchai, S., Wanaree, W. and Sawasd, T. "Performance of M-PSK in Mobile Satellite Communication Over Combined Ionosphere Scintillation and Flat Fading Channels with MRC Diversity", IEEE Transactions on Wireless Communications, (2009), Vol. 8, No. 7.

[27] Proakis, J. G, “Digital Communications”, McGraw-Hill companies, incorporation. International Edition, 2001.

[28] Rappaport, T.S., "Wireless Communication Principles and Practice" $2^{\text {nd }}$ edition Prentice Hall of India Private limited view Delhi, (2002), pp1-526.

[29] Simeon M.K.and Alouini, M. S, “Digital Communications Over Fading Channels",2 ${ }^{\text {nd }}$ edition, John Wiley and Sons, Incorporation, Hoboken, New Jersey, (2005), Pp1 - 523.

[30] Stewart, K.A., Labedz, and sohrabi K., "Wideband channel measurements at 900MHZ" in Proc. IEEE Vehicular Technology Conf. (VTC'95), Chicago IL, (1995) pp 236-240.

[31] Tang L. and Hongbo Z., "Analysis of Nakagami Fading channel with MATLAB” Asifat Pacific Conference on Environmental Electromagnetic CEEM Hang zhou, China. (2003), Pp490 - 494.

[32] Tse, D., and Viswanath, P., "Fundamentals of Wireless Communication" $1^{\text {st }}$ Edition; Cambridge University Press, New York. (2005).

[33] Ye, Z. and Satorius "Channel modeling and simulation for mobile user objective system (MUOS) - part 1: flat scintillation and fading", in proceeding IEEE ICC, (2003), 5(1):3508-3510. 